\title{
Determination of Structural Parameters by EXAFS Analysis of Some Co Complexes
}

\author{
A. Mishra $^{a, *}$, N. PARSAI ${ }^{b}$ AND N. DAgAONKAR ${ }^{c}$ \\ ${ }^{a}$ School of Physics, Devi Ahilya University, Indore (M.P.) 452001, India \\ ${ }^{b}$ Department of Physics, Sanghvi Innovative Academy, Indore (M.P.), India \\ ${ }^{c}$ Physics Department, Govt. P.G. College, Dhar 457-001, India
}

(Received October 26, 2009; in fianl form December 7, 2009)

\begin{abstract}
In this paper we deal with a relatively simpler method for the Fourier analysis of EXAFS data and illustrate this by a detailed analysis of the data for cobalt metal foil and cobalt complexes of isoxezol series. The Fourier analysis is useful in extracting distances and information about the phase shifts, the amplitudes, the Debye-Waller factors and the mean free paths. Here we have determined interatomic distances, mean square deviation and inelastic mean free path from the Fourier transform of EXAFS data for cobalt metal foil and cobalt complexes of isoxezol series by MathCAD programming.
\end{abstract}

PACS numbers: 61.05.cj

\section{Introduction}

Extended X-ray absorption fine structure (EXAFS) spectroscopy provides structural information about a sample by way of the analysis of its X-ray absorption spectrum. In order to extract structural information from experimental spectra, a simple analytical expression that relates the EXAFS signal to the structural parameters is required. An EXAFS analytical expression like the one suggested by Stern [1] is

$$
\begin{gathered}
\chi(k)=\frac{m}{4 \pi \hbar^{2} k} \sum_{j} \frac{N_{j}}{R_{j}^{2}} t_{j}(2 k) \exp \left(-2 R_{j} / \lambda\right) \\
\times \sin 2\left(k R_{j}+\delta_{j}(k)\right) \exp \left(-2 k^{2} \sigma_{j}^{2}\right),
\end{gathered}
$$

where $k$ is the electron wave vector, $N_{j}$ is the number of atoms in the $j$-th coordination shell, $R_{j}$ is the average radial distance to the $j$-th atom, $t_{j}(2 k)$ is the backscattering matrix element encountered by the electrons, $\lambda$ is the mean free path of the electron, the 2nd exponential containing $\sigma_{j}^{2}$ is a Debye-Waller-type term where $\sigma_{j}$ is the root mean square deviation of the actual position of the atoms in the $j$-th shell about $R_{j}$, and $\delta_{j}(k)$ is a phase shift approximately linear in $k$ over the EXAFS energy range with a negative slope [2] i.e.

$$
\delta_{j}=-\alpha_{j} k+\beta_{j}
$$

where $\alpha_{j}$ and $\beta_{j}$ are constants.

Various structural parameters can be extracted by an approximate Fourier analysis of the normalized EXAFS term $\chi(k)$ [3]. From $\chi(k)$ a radial function $\phi_{n}(R)$ can be

\footnotetext{
* corresponding author; e-mail: neetumandloi@rediffmail.com
}

derived [4] given by

$$
\phi_{n}(R)=\frac{1}{\sqrt{2 \pi}} \int_{k_{\min }}^{k_{\max }} k^{n} \chi(k) \exp (2 \mathrm{i} k R) \mathrm{d} k
$$

where $n$ is usually either 1 or 3 , and $k_{\min }$ and $k_{\max }$ are the minimum and maximum values of $k$, respectively. In addition to the interatomic distance $R$, the mean square deviation $\sigma^{2}$ of $R$, and the number of atoms $N$ at $R$ can be extracted from an EXAFS spectrum.

In this paper the physical parameters from the $K$-edge data of cobalt metal foil and cobalt(II) complexes of isoxezol series are obtained theoretically using MathCAD programming. Previously these parameters are obtained for copper metal foil and copper(II) complexes of isoxezol series $[5,6]$. The physical parameters for EXAFS are obtained by an approximate Fourier analysis of the data.

\section{Methodology}

EXAFS data for cobalt metal foil and cobalt(II) complexes of isoxezol series are generated theoretically using Eq. (1) in MathCAD programming from experimental data [7]. The experimental data were obtained on performing the experiment on conventional X-ray fixed target source. The values of $(R-\alpha)$ and $\beta$ determined experimentally using LSS [8] method are used in Eq. (1) to generate EXAFS data theoretically. Theoretical graphs are plotted for $\chi(k)$ versus $k$ as shown in Fig. 1 over the range of $k$ from 2.7 to $15.8 \AA^{-1}$. The magnitude of the radial function $\phi_{3}(R)$ is obtained from Eq. (3), for cobalt metal foil and cobalt(II) complexes of isoxezol series, and it is shown in Fig. 2. This magnitude of the radial function $\phi_{3}(R)$ is used to calculate structural data for cobalt 
metal and cobalt(II) complexes of isoxezol series and it is tabulated in Table I.

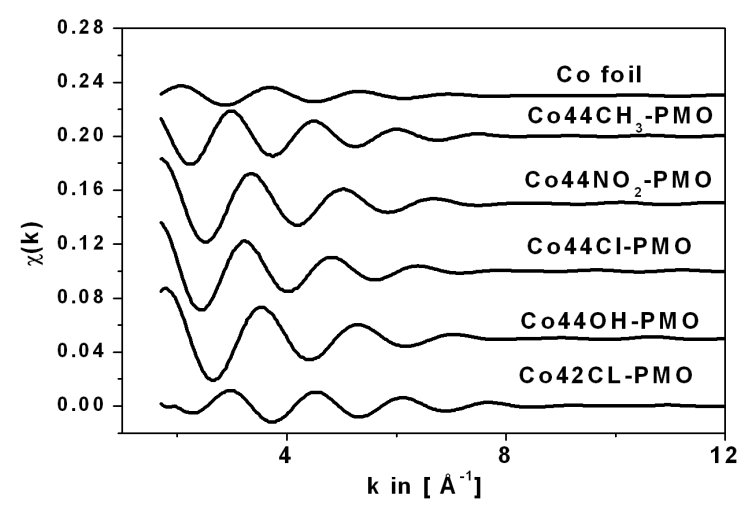

Fig. 1. Normalized EXAFS, $\chi(k)$ versus $k\left[\AA^{-1}\right]$ (theoretical) for cobalt metal foil and cobalt(II) complexes.

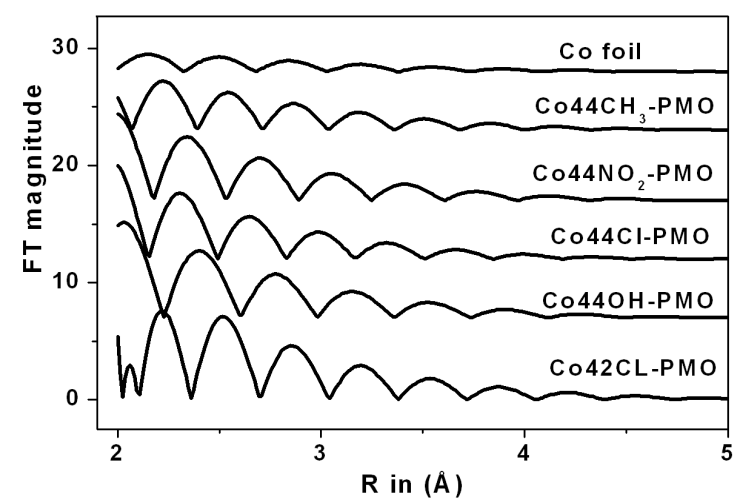

Fig. 2. Magnitude of Fourier transform $\Phi_{3}(R)$ versus $R[\AA]$ (theoretical) for cobalt metal foil and cobalt(II) complexes.

On putting the value of $\delta_{j}(k)$ from Eq. (2) into Eq. (3), it can be written as

$$
\begin{aligned}
& \phi_{3}(R)=\frac{m}{4 \pi \hbar^{2}} \sum \frac{N_{j}}{R_{j}^{2}} \mathrm{e}^{-2 R_{j} / \lambda} \int k^{2} t_{j}(2 k) \\
& \quad \times \mathrm{e}^{-2 \sigma_{j}^{2} k^{2}} \mathrm{e}^{2 \mathrm{i} k R_{j}} \sin 2\left(k\left(R_{j}-\alpha_{j}\right)+\beta_{j}\right) \mathrm{d} k .
\end{aligned}
$$

The value of mean free path $(\lambda)$ can be obtained from Eq. (4) by plotting the $\ln \left(\left(A_{j} R_{j}^{2} / N_{j}\right) /\left(A_{1} R_{1}^{2} / N_{1}\right)\right)$ versus $R_{j}$, where $A_{j}$ is the amplitude of the peak of the $j$-th shell. If $\sigma_{j}$ and $t_{j}$ are independent of $j$, then such a plot should be a straight line, whose slope is $-2 / \lambda$. Such a plot is shown in Fig. 3 for cobalt metal foil. The plot is nearly linear.

Deviation from linearity can be understood by the fact that theoretically $\sigma_{j}$ is expected to be different for various $j$. Similarly, mean path for cobalt complexes of isoxezol series are calculated. The value of $\lambda$ for cobalt metal and cobalt(II) complexes of isoxezol series calculated from slope is shown in Table II.

If $\sigma_{j}$ is the same for all shells and independent of $j$, the standard normalized EXAFS term has the simple form
TABLE I

Theoretically calculated structural data for cobalt metal foil and the cobalt(II) complexes. $R_{j}$ is the value of radial distance in Fig. 2 for peak positions of magnitude of the Fourier transform and $A_{j}$ is the corresponding value of magnitude of the Fourier transform and

\begin{tabular}{|c|c|c|c|c|}
\hline Complexes & $i$ & $R_{j}[\AA]$ & $A_{j}$ & $B_{j} / B_{1}$ \\
\hline \multirow[t]{6}{*}{ Co foil } & 1 & 2.17 & 2.95 & 1 \\
\hline & 2 & 2.49 & 2.54 & 0.95 \\
\hline & 3 & 2.86 & 1.83 & 0.80 \\
\hline & 4 & 3.19 & 1.24 & 0.60 \\
\hline & 5 & 3.53 & 0.77 & 0.44 \\
\hline & 6 & 3.90 & 0.46 & 0.26 \\
\hline \multirow[t]{6}{*}{ Co42Cl-PMO } & 1 & 2.52 & 7.07 & 1 \\
\hline & 2 & 2.84 & 4.60 & 0.82 \\
\hline & 3 & 3.21 & 2.90 & 0.66 \\
\hline & 4 & 3.53 & 1.77 & 0.49 \\
\hline & 5 & 3.87 & 1.06 & 0.35 \\
\hline & 6 & 4.21 & 0.60 & 0.23 \\
\hline \multirow[t]{6}{*}{ Co44OH-PMO } & 1 & 2.04 & 8.09 & 1 \\
\hline & 2 & 2.39 & 5.66 & 0.96 \\
\hline & 3 & 2.77 & 3.70 & 0.84 \\
\hline & 4 & 3.14 & 2.23 & 0.65 \\
\hline & 5 & 3.51 & 1.29 & 0.47 \\
\hline & 6 & 3.91 & 0.67 & 0.30 \\
\hline \multirow[t]{6}{*}{$\mathrm{Co} 44 \mathrm{NO}_{2}-\mathrm{PMO}$} & 1 & 2.34 & 5.47 & 1 \\
\hline & 2 & 2.68 & 3.64 & 0.87 \\
\hline & 3 & 3.03 & 2.33 & 0.71 \\
\hline & 4 & 3.41 & 1.40 & 0.54 \\
\hline & 5 & 3.76 & 0.81 & 0.38 \\
\hline & 6 & 4.1 & 0.42 & 0.23 \\
\hline \multirow[t]{6}{*}{$\mathrm{Co}_{44 \mathrm{CH}_{3}-\mathrm{PMO}}$} & 1 & 2.22 & 5.2 & 1 \\
\hline & 2 & 2.54 & 3.23 & 0.81 \\
\hline & 3 & 2.86 & 2.25 & 0.71 \\
\hline & 4 & 3.17 & 1.53 & 0.59 \\
\hline & 5 & 3.51 & 1.00 & 0.48 \\
\hline & 6 & 3.83 & 0.61 & 0.34 \\
\hline \multirow[t]{6}{*}{ Co44Cl-PMO } & 1 & 2.31 & 5.57 & 1 \\
\hline & 2 & 2.64 & 3.60 & 0.84 \\
\hline & 3 & 2.98 & 2.33 & 0.69 \\
\hline & 4 & 3.32 & 1.36 & 0.50 \\
\hline & 5 & 3.67 & 0.80 & 0.36 \\
\hline & 6 & 3.99 & 0.40 & 0.21 \\
\hline
\end{tabular}
$B_{j} / B_{1}=N_{1} A_{j} R_{j}^{2} / N_{j} A_{1} R_{1}^{2}$.

$$
\chi(k)=\chi 1(k) \mathrm{e}^{-2 k^{2} \sigma^{2}},
$$

where

$$
\begin{aligned}
& \chi 1(k)=\frac{m}{4 \pi \hbar^{2} k} \sum \frac{N_{j}}{R_{j}^{2}} \mathrm{e}^{-2 R_{j} / \lambda} t_{j}(2 k) \mathrm{e}^{2 \mathrm{i} k R_{j}} \\
& \quad \times \sin 2\left(k\left(R_{j}-\alpha_{j}\right)+\beta_{j}\right) .
\end{aligned}
$$




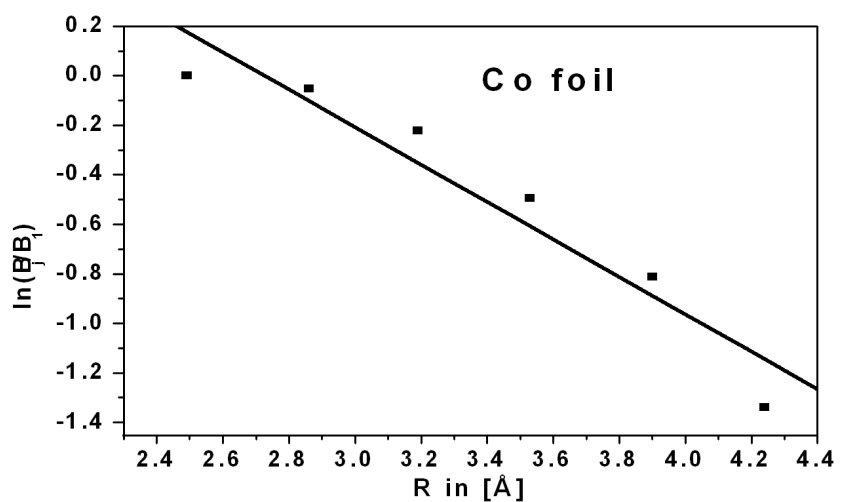

Fig. 3. $\ln \left(B_{j} / B_{1}\right)$ versus $R[\AA]$ for cobalt metal foil.

TABLE II

Theoretically calculated mean free path $(\lambda)$ for cobalt metal foil and cobalt(II) complexes.

\begin{tabular}{c|c|c}
\hline \hline No. & Complexes & Mean free path $\lambda[\AA]$ \\
\hline 01 & Co foil & 2.66 \\
02 & Co 42Cl-PMO & 2.35 \\
03 & Co44OH-PMO & 2.5 \\
04 & Co44Cl-PMO & 2.22 \\
05 & Co44NO 2 -PMO & 2.5 \\
06 & Co44CH 3 -PMO & 3.22
\end{tabular}

Thus taking the ratio of $\chi(k)$ and $\chi 1(k)$, it is possible to determine

$$
\sigma^{2}=\left(\frac{1}{2 k^{2}}\right) \ln \frac{\chi(k)}{\chi 1(k)}
$$

by plotting the $\ln \chi(k) / \chi 1(k)$ on the right hand side of Eq. (5) as a function of $k^{2}$ and determining the slope of the resulting straight line. A plot of $\ln \chi(k) / \chi 1(k)$ versus $k^{2}$ is shown in Fig. 4, using EXAFS data. This figure has shown a straight line. The slope of the straight line gives the value of $\sigma^{2}=0.0043 \AA^{2}$. This value is

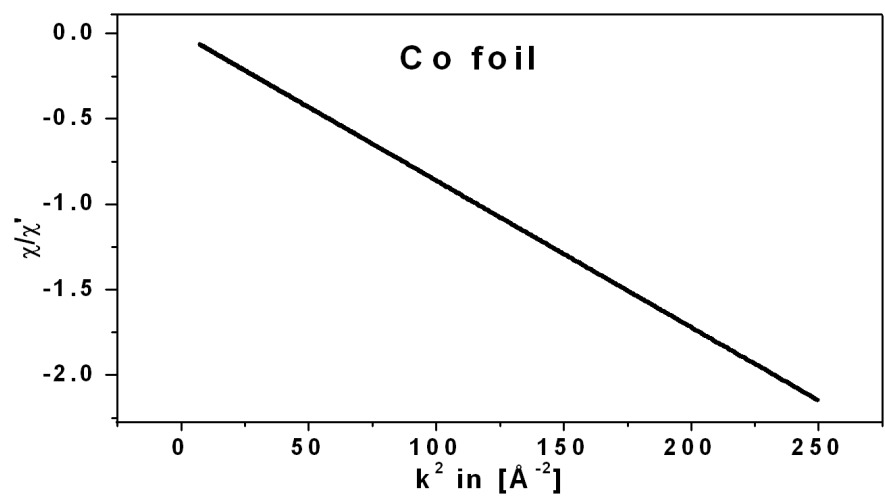

Fig. 4. $\ln \chi(k) / \chi 1(k)$ versus $k^{2}\left[\AA^{-2}\right]$ for cobalt metal foil. in reasonable agreement with $\sigma^{2}$ determined from X-ray measurements of the Debye-Waller factor for cobalt using other known standard techniques.

\section{Conclusion}

In this paper, we have derived a number of physical parameters by the Fourier analysis of EXAFS data of cobalt metal foil and cobalt(II) complexes of isoxezol series, obtained using the theoretical expression of EXAFS. The values of the parameters, mean square deviation $\left(\sigma^{2}\right)$ and mean free path $(\lambda)$ calculated using MathCAD programming for theoretical EXAFS data of cobalt metal foil are in a good agreement with the values of these parameters determined from X-ray measurements of the Debye-Waller factor by Noronha [9].

\section{References}

[1] E.A. Stern, Phys. Rev. B 10, 3027 (1974).

[2] B.K. Teo, P.A. Lee, J. Am. Chem. Soc. 101, 2815 (1979).

[3] D.E. Sayers, E.A. Stern, F.W. Lytle, Phys. Rev. Lett. 27, 1204 (1971).

[4] E.A. Stern, D.E. Sayers, F.W. Lytle, Phys. Rev. B 11, 4836 (1975).

[5] A. Mishra, N. Parsai, N. Dagaokar, Int. J. Mod. Phys. B, accepted for publication.

[6] A. Mishra, N. Parsai, N. Dagonkar, Indian J. Pure Appl. Phys. 47, 337 (2009).

[7] N. Dagaokar, Ph.D. Thesis, Devi Ahilya University, Indore 2004, p. 88.

[8] F.W. Lytle, D.E. Sayers, E.A. Stern, Phys. Rev. B 11, 4825 (1975).

[9] F.B. Noronha, Braz. J. Chem. Eng. 16, 309 (1999). 